\title{
ON COMPLETE RIEMANNIAN MANIFOLDS WITH COLLAPSED ENDS
}

\author{
ZHONGMIN SHEN
}

\begin{abstract}
We show that if a complete open manifold with bounded curvature and sufficiently small ends, then each end is an infranilend. Conversely, an open manifold with finitely many infranilends admits a complete metric with bounded curvature and arbitrarily small ends.
\end{abstract}

1. Introduction. It is well known that if a complete open manifold $M$ of finite volume has bounded negative sectional curvature, i.e.

$$
-\Lambda_{2} \leq \sec (M) \leq-\Lambda_{1},
$$

where $\Lambda_{i}$ are positive constants, then $M$ has finite topological type (see [G1]). In particular, $M$ has finitely many ends. Moreover, each end collapses, i.e., for any end $E$ and any point $p \in M$,

$$
\lim _{r \rightarrow \infty} \operatorname{diam}(E \cap S(p, r))=0,
$$

where $S(p, r)=\{x \in M ; d(p, x)=r\}$ denotes the geodesic sphere of radius $r$ around $p$. Further, each end is topologically of the form $N \times(0, \infty)$ for some infranilmanifold $N$. See [E] and [Sc] for details. See also [K] in the case $\Lambda_{2} / \Lambda_{1}<4$.

An open manifold $M$ is said to have $N$ ends, if there is a compact subset $K$ such that for any compact subset $K \subset K^{\prime} \subset M, M \backslash K^{\prime}$ contains exactly $N$ unbounded components. Simply we call any such component an end of $M$.

In [Sh] we studied complete open Riemannian manifolds $M$ with sectional curvature bounded from below and small ends. In order to state the result, we need to introduce some notations. For $r>0$, the connected components, $\Sigma$, of $\partial(M \backslash \overline{B(p, r)})$, are called the boundary components, where $B(p, r)$ denotes the open geodesic ball of radius $r$ around $p$. Following [C] (compare [AG]), we define the essential diameter $\mathscr{D}(p, r)$ at distance $r$ from $p$ by

$$
(p, r)=\sup _{\Sigma} \operatorname{diam}(\Sigma),
$$

where the supremum is taken over all boundary components $\Sigma$ of $M \backslash \overline{B(p, r)}$ with $\Sigma \cap R(p, r) \neq \varnothing$, where $R(p, r)=\{\gamma(r) ; \gamma$ is a ray from $p\} \subset S(p, r)$. Notice that in the definition of $\mathscr{D}(p, r)$ we do not 
assume that $M$ has finitely many ends. In [Sh] we prove the following

TheOREM 1 ([Sh]). Let $M$ be complete with $\sec (M) \geq-1$. Suppose that

$$
\varlimsup_{r \rightarrow \infty} \mathscr{D}(p, r)<\ln 2 .
$$

Then $M$ is homeomorphic to the interior of a compact manifold with boundary. In particular, $M$ has finitely many ends.

We do not know what is the best constant on the right side of (1). This theorem is proved by applying the Morse theory to the distance function $d_{p}(x)=d(p, x)$. Of course, $d_{p}$ is not of $C^{1}$ in general. But we still have a notion of critical points of $d_{p}$. We say a point $q$ is a critical point in the sense of Grove-Shiohama [GS] if for any vector $v \in T_{q} M$, there is a minimal geodesic $\sigma$ from $q$ to $p$, making an angle $\angle(v, \dot{\sigma}(0)) \leq \frac{\pi}{2}$. For the further discussion in $\S 2$, we would like to outline the proof of Theorem 1 here (see [Sh] and [G] for details). Suppose $M$ is as in Theorem 1. Let $e_{p}$ denote the excess function on $M$, which is defined by $e_{p}(x)=\lim _{r \rightarrow+\infty} d_{p}(x)+d(x, S(p, r))-r$. It follows from Toponogov's comparison theorem that if $q \in S(p, r)$ is a critical point of $d_{p}$, then

$$
e_{p}(q) \geq \ln \frac{e^{r}}{\cosh r}
$$

Let $\Sigma$ be any boundary component of $M \backslash \overline{B(p, r)}$ with $\Sigma \cap R(p, r) \neq$ $\varnothing$. An elementary argument shows that for any $x \in \Sigma$,

$$
e_{p}(x) \leq \operatorname{diam}(\Sigma) \leq \mathscr{D}(p, r) .
$$

Notice that $\ln \left(e^{r} / \cosh r\right) \rightarrow \ln 2$ as $r \rightarrow+\infty$. Thus there is a large $R_{0}$ such that if $r \geq R_{0}, \Sigma$ contains no critical points of $d_{p}$. Let $E$ be an unbounded component of $M \backslash \overline{B\left(p, R_{0}\right)}$, and let $\gamma$ be a ray from $p$ such that $\gamma\left(R_{0}, \infty\right) \subset E$. Denote by $\Sigma_{r}$ the boundary component of $M \backslash \overline{B(p, r)}$ with $\gamma(r) \in \Sigma_{r}, r \geq R_{0}$. Since all $\Sigma_{r}, r \geq R_{0}$, contain no critical points of $d_{p}$, one can show that all $\Sigma_{r}$ are homeomorphic, and $E$ is homeomorphic to $\Sigma_{R_{0}} \times\left(R_{0}, \infty\right)$. Notice that $M \backslash \overline{B\left(p, R_{0}\right)}$ has finitely many unbounded components. Thus $M$ has finite topological type in the sense of Theorem 1. In this case, it is also easy to see

$$
\mathscr{D}(p, r)=\max _{E} \operatorname{diam}(E \cap S(p, r)), \quad r \geq R_{0},
$$


where the maximum is taken over all unbounded components of $M \backslash \overline{B\left(p, R_{0}\right)}$. Readers can also refer to [C] for further discussion.

In this paper we will study the structure of small ends for complete open manifolds with bounded sectional curvature. Riemannian manifolds under consideration may have infinite volume and are not required to be negatively curved.

THEOREM 2. Let $M$ be a complete open $n$-manifold with $p \in M$ fixed. Suppose that $|\sec (M)| \leq 1$. There is a small $\varepsilon(n)>0$, such that if

$$
\varlimsup_{r \rightarrow \infty} \mathscr{D}(p, r)<\varepsilon(n),
$$

then there is a compact subset $K \subset M$, such that each unbounded component of $M \backslash K$ is diffeomorphic to $N \times(0, \infty)$ for some infranilmanifold $N$.

A manifold $N$ is called an infranilmanifold if it is diffeomorphic to a compact space $G / \Gamma$, where $G$ is a nilpotent Lie group and $\Gamma$ is a discrete group of affine transformations of $G$ satisfying $[\Gamma: G \cap \Gamma]<$ $\infty$. Here we have put the left invariant connection $D$ on $G$ for which left invariant vector fields are parallel and $G$ is regarded as a group of affine transformations on $G$ by left translations (see [R]). An end of an open manifold, which is diffeomorphic to $N \times(r, \infty)$ for some infranilmanifold, is called an infranilend.

Suppose $M$ is an open $n$-manifold with finitely many infranilends. A natural question is whether or not $M$ admits a complete metric $g$ such that $|\sec (M, g)| \leq 1$ and each one $E \cong N \times(0, \infty)$ collapses, i.e.

$$
\varlimsup_{r \rightarrow \infty} \operatorname{diam}(N \times\{r\})=0 .
$$

The answer is affirmative.

THEOREM 3. If $M$ is an open manifold with finitely many infranilends, then $M$ admits a complete Riemannian metric $g$ satisfying $|\sec (M)| \leq 1$ and (3) for each end $E \cong N \times(0, \infty)$.

The construction of the metric is not trivial, and is given in $\S 3$. One needs to know precisely the structure of an infranilmanifold. Our construction is inspired by [W1].

2. Proof of Theorem 2. The proof of Theorem 2 is simple. We are going to apply a theorem of Fukaya [F1] and [F2] to our case. 
For two metric spaces $X, Y$ and $\varepsilon>0$, a map $h: X \rightarrow Y$ is said to be an $\varepsilon$-Hausdorff approximation if for any points $x_{1}, x_{2} \in X$,

$$
\left|d\left(x_{1}, x_{2}\right)-d\left(h\left(x_{1}\right), h\left(x_{2}\right)\right)\right|<\varepsilon
$$

and the image of $h, h(X) \subset Y$, is $\varepsilon$-dense in $Y$. Let $M_{1}^{n}, M_{2}^{m}, m \leq$ $n$, be complete with $\left|\sec \left(M_{1}\right)\right| \leq 1,\left|\sec \left(M_{2}\right)\right| \leq 1$ and $\operatorname{inj}\left(M_{2}\right) \geq i_{0}$. Fukaya's theorem says that there is a small number $\varepsilon=\varepsilon\left(n, i_{0}\right)$ so that if $h: M_{1} \rightarrow M_{2}$ is an $\varepsilon$-Hausdorff approximation, then there is a fibration $f: M_{1} \rightarrow M_{2}$ such that $f^{-1}(y), y \in M_{2}$, is an infranilmanifold. Fukaya's theorem is an important generalization of Gromov's result [G2] and $[\mathbf{R}]$ on almost flat manifolds.

Now we let $M$ be a complete open $n$-manifold with $p \in M$ fixed. First of all we only assume that

$$
\mathscr{D}(p, r)<\delta, \quad r \geq R_{0},
$$

for some $\delta<\ln 2$. As mentioned in $\S 1$, taking a larger number $R_{0}$ if necessary, one can show that any unbounded component, $E$, of $M \backslash \overline{B\left(p, R_{0}\right)}$ is homeomorphic to $N \times(0, \infty)$, where $N$ is a compact manifold without boundary (see [Sh] for details). Furthermore, by (2)

$$
\mathscr{D}(p, r)=\max _{E} \operatorname{diam}(E \cap S(p, r))<\delta, \quad r \geq R_{0},
$$

where the maximum is taken over all unbounded components, $E$, of $M \backslash \overline{B\left(p, R_{0}\right)}$.

Fix an unbounded component $E$ of $M \backslash \overline{B\left(p, R_{0}\right)}$. Define

$$
\begin{aligned}
\pi: E & \rightarrow\left(R_{0}, \infty\right), \\
x \in E \cap S(p, r) & \rightarrow r \in\left(R_{0}, \infty\right) .
\end{aligned}
$$

It is easy to see by (4) that for any $x_{1}, x_{2} \in E$,

$$
\left|d\left(x_{1}, x_{2}\right)-\right| \pi\left(x_{1}\right)-\pi\left(x_{2}\right)||<2 \delta .
$$

Thus $\pi$ is an $2 \delta$-Hausdorff approximation.

Although Fukaya's theorem is stated for complete manifolds, his argument can be carried over to our case. Let $E_{1}=\{x \in E, d(x, \partial E)>$ $1\}$. By [F1] and [F2] there is a small number $\varepsilon(n)>0$ such that if (5) holds for some $\delta<\varepsilon(n)$, then there is an open neighborhood $U, E_{1} \subset U \subset E$, and a fibration $f: U \rightarrow\left(R_{1}, \infty\right), R_{1} \geq R_{0}$, with fibres $f^{-1}(r)$ diffeomorphic to an infranilmanifold $N$ by $\phi_{r}$. One then defines a diffeomorphism $\phi: U \rightarrow N \times\left(R_{1}, \infty\right)$ by $\phi(x)=\left(\phi_{r}(x), r\right)$ for $x \in f^{-1}(r)$.

One can also follow [CFG, §2] to construct the above fibration $f$ by the center of mass techniques. This completes the proof. 
Let $M$ be a complete $n$-manifold with $N$ ends. Let $E_{1}, \ldots, E_{N}$ denote all unbounded connected components of $M \backslash K$, where $K$ is some compact subset. Let $p \in M$. Set

$$
\operatorname{diam}(p, M)=\varlimsup_{r \rightarrow \infty} \max _{1 \leq i \leq N} \operatorname{diam}\left(E_{i} \cap S(p, r)\right) .
$$

Clearly, $\operatorname{diam}(p, M)$ is independent of $K$. The following corollary is a direct consequence of the above argument.

COROLlaRY 1. Let $M$ be complete $n$-manifold with finitely many ends. Suppose that $|\sec (M)| \leq 1$. There is a small $\varepsilon(n)$, if for some point $p \in M$,

$$
\operatorname{diam}(p, M)<\varepsilon(n),
$$

then there is a compact subset $K$ such that each unbounded component $E$ of $M \backslash K$ is diffeomorphic to $N \times(0, \infty)$, where $N$ is an infranilmanifold.

3. Construction of the metrics. Let $M$ be an open manifold with finitely many infranilends, say, $E_{1}, \ldots, E_{N}$, which are unbounded connected components of $M \backslash K$ for some compact subset $K$. By definition, each end $E_{i}$ is diffeomorphic to $N_{i} \times(0, \infty)$ for some infranilmanifold $N_{i}$. Fix an end $E \cong N \times(0, \infty)$. In order to construct a complete metric on $M$ satisfying (3) in Theorem 3, it suffices to construct a metric $g$ on $E \cong N \times(0, \infty)$ such that $|\sec (E, g)| \leq 1$ and (3) holds.

Recall that $N$ is an infranilmanifold if $N=G / \Gamma$, where $G$ is a nilpotent Lie group and $\Gamma$ is a discrete group of affine transformations of $G$ satisfying $[\Gamma, G \cap \Gamma]<\infty$. Here we have put the left invariant connection $D$ on $G$ for which left invariant vector fields are parallel, and $G$ is regarded as a group of affine transformations on $G$ by left translations. Let $L$ denote the Lie algebra of $G$, the space of leftinvariant vector fields on $G$. One has the following stratification

$$
L=L_{0} \supset L_{1} \supset \cdots \supset L_{k} \supset L_{k+1}=0
$$

where $L_{i+1}=\left[L, L_{i}\right]$. Notice that $H:=\Gamma /(G \cap \Gamma)$ acts on $L$ and preserves the stratification (6). One can choose an $H$-invariant inner product $\langle,\rangle_{0}$ on $L$. Let $F_{i}=\left\{X \in L_{i},\langle X, Y\rangle_{0}=0, Y \in L_{i+1}\right\}$. Then $L=F_{0} \oplus \cdots \oplus F_{k}$. One can define an $H$-invariant inner product $\langle,\rangle_{r}$ on $L$ by

$$
\langle X, Y\rangle_{r}=h_{i}(r)^{2}\langle X, Y\rangle_{0}, \quad X, Y \in F_{i},
$$


and $\langle X, Y\rangle_{r}=0$ if $X \in F_{i}, Y \in F_{j}, i \neq j$, where $h_{i}$ are some positive functions which are to be determined later. $\langle,\rangle_{r}$ defines an $H$-invariant metric on $G$, which is then $\Gamma$-invariant. Hence it induces a Riemannian metric $g_{r}$ on $G / \Gamma$. There is a bound $C$ depending only on $G$ and $\langle,\rangle_{0}$ such that

$$
\|[X, Y]\|_{0} \leq C\|X\|_{0}\|Y\|_{0}
$$

for all $X, Y \in L$. For a left-invariant vector $X \in L$, we denote it by $Z=\sum_{i} Z_{i}$, where $Z_{i}$ denotes the component of $Z$ in $F_{i}$. Then for any $X, Y \in L$, one has

$$
\left\|[X, Y]_{i}\right\|_{0} \leq\|[X, Y]\|_{0} \leq C\|X\|_{0}\|Y\|_{0} .
$$

We choose $h_{i}$ in such a way that the following inequalities hold:

$$
\sum_{s=i+1}^{k} h_{s}(r) \leq h_{i}(r)^{2} \leq h_{i-1}(r)^{2} .
$$

Then for $X=\sum_{i=0}^{k} X_{i}$ and $Y=\sum_{i=0}^{k} Y_{i}$,

(8) $\|[X, Y]\|_{r}=\left\|\sum_{i j}\left[X_{i}, Y_{j}\right]\right\|_{r} \leq \sum_{i j}\left\|\left[X_{i}, Y_{j}\right]\right\|_{r}$

$$
\begin{aligned}
& \leq \sum_{i j} \sum_{s>\max (i j)} h_{s}(r)\left\|\left[X_{i} . Y_{j}\right]_{s}\right\|_{0} \\
& \leq C \sum_{i j} \sum_{s>\max (i j)} h_{s}(r)\left\|X_{i}\right\|_{0}\left\|_{Y_{j}}\right\|_{0} \\
& \leq C \sum_{i j} h_{i}(r) h_{j}(r)\left\|X_{i}\right\|_{0}\left\|Y_{j}\right\|_{0} \leq(k+1) C\|X\|_{r}\|Y\|_{r} .
\end{aligned}
$$

Let $\widehat{\nabla}$ denote the Levi-Civita connection of $g_{r}$, and $\widehat{R}$ its curvature tensor. It follows from (8) and formulas in [CE, Proposition 3.18], that

$$
\begin{aligned}
\left\|\widehat{\nabla}_{X} Y\right\|_{r} & \leq \frac{3}{2}(k+1) C, \\
\|\widehat{R}(X, Y) Z\|_{r} & \leq 6(k+1)^{2} C^{2}
\end{aligned}
$$

for any orthonormal left-invariant vector fields $X, Y$ and $Z$ in $L$ with respect to $\langle,\rangle_{r}$.

We define a warped product metric $g$ on $E=N \times(0, \infty)$ by

$$
g=g_{r} \oplus d r^{2} \text {. }
$$


Let $H=\frac{\partial}{\partial r}$ and $X, Y, Z$, etc. be left-invariant vector fields on $G$. Let $\nabla$ denote the Levi-Civita connection of $g$. Then it is easy to get the following

$$
\begin{aligned}
& \nabla_{H} H=0, \\
& \nabla_{H} X=\nabla_{X} H=\frac{h_{i}^{\prime}(r)}{h_{i}(r)} X, \\
& \nabla_{X} Y=\widehat{\nabla}_{X} Y-\frac{h_{i}^{\prime}(r)}{h_{i}(r)}\langle X, Y\rangle_{r} H
\end{aligned}
$$

for any left-invariant vector fields $X \in F_{i}$ and $Y \in F_{j}$.

We now take $h_{i}(r)=e^{-\alpha_{i}(r+1)}, \alpha_{i}=2^{i}-1$. Then (7) holds. It is easy to see that $g$ has bounded curvature on $E \cong N \times(0, \infty)$. More precisely, if

$$
\left|\frac{h_{i}^{\prime}(r)}{h_{i}(r)}\right| \leq \alpha_{k}, \quad\left|\frac{h_{i}^{\prime \prime}(r)}{h_{i}(r)}\right| \leq \alpha_{k}^{2},
$$

then by (9) (10) there is constant $K(k, C)$ such that

$$
\begin{aligned}
&\|R(X, H) H\| \leq K(k, C), \\
&\|R(X, Y) H\| \leq K(k, C), \\
&\|R(X, H) Y\| \leq K(k, C), \\
&\|R(X, Y) Z\| \leq K(k, C)
\end{aligned}
$$

for any orthonormal left-invariant vector fields $X \in F_{i}, Y \in F_{j}$ and $Z \in F_{m}$.

In particular, one has (compare [W2])

$$
\begin{aligned}
& g(R(X, H) H, X)=-\frac{h_{i}^{\prime \prime}(r)}{h_{i}(r)}, \\
& g(R(X, Y) Y, X)=g_{r}(\widehat{R}(X, Y) Y, X)-\frac{h_{i}^{\prime}(r) h_{j}^{\prime}(r)}{h_{i}(r) h_{j}(r)}
\end{aligned}
$$

for orthonormal left-invariant vector fields $X \in F_{i}, Y \in F_{j}$.

Observe that $g_{r} \leq e^{-2(r+1)} g_{0}$. One concludes that

$$
\operatorname{diam}\left(N \times\{r\}, g_{r}\right) \leq e^{-r-1} \cdot \operatorname{diam}\left(N, g_{0}\right) \rightarrow 0
$$

as $r \rightarrow \infty$. This completes the proof.

ReMARK. Taking $h_{i}(r)=e^{-\alpha_{t}(r+1)}, \alpha_{i}=\delta \cdot 2^{i}-1$, one can make the curvature negatively pinched on each end, provided that $\delta$ is sufficiently large. 


\section{REFERENCES}

[AG] U. Abresch and D. Gromoll, On complete manifolds with nonnegative Ricci curvature, J. Amer. Math. Soc., 3 (1990), 355-374.

[C] J. Cheeger, Critical points of distance functions and applications to geometry, preprint (1990).

[CE] J. Cheeger and D. Ebin, Comparison Theorems in Riemannian Geometry, North-Holland, Amsterdam, 1975.

[CFG] J. Cheeger, K. Fukaya and M. Gromov, Nilpotent structure and invariant metrics on collapsed manifolds, preprint.

[E] P. Eberlein, Lattices in spaces of nonpositive curvature, Ann. of Math., 111 (1980), 435-476.

[F1] K. Fukaya, Collapsing Riemannian manifolds to ones of lower dimension, J. Differential Geom., 25 (1987), 137-156.

[F2] _ Collapsing Riemannian manifolds to ones of lower dimension, II, J. Math. Soc. Japan, 41 (1989), 333-356.

[G1] M. Gromov, Manifolds of negative curvature, J. Differential Geom., 13 (1978), 223-230.

[G2] _ Almost flat manifolds, J. Differential Geom., 13 (1978), 231-242.

[G] K. Grove, Critical point theory for distance functions, preprint.

[GS] K. Grove and K. Shiohama, A generalized sphere theorem, Ann. of Math., 106 (1977), 201-211.

[K] M. Kanai, A pinching theorem for cusps of negatively curved manifolds with finite volume, preprint (1990).

[R] E. Ruh, Almost flat manifolds, J. Differential Geom., 17 (1982), 1-14.

[Sc] V. Schroeder, Finite volume and fundamental group on manifolds of negative curvature, J. Differential Geom., 20 (1984), 175-183.

[Sh] Z. Shen, On complete manifolds of nonnegative kth-Ricci curvature, Trans. Amer. Math. Soc., 338 (1993), 289-310.

[W1] G. Wei, Examples of complete manifolds of positive Ricci curvature with nilpotent isometry groups, Bull. Amer. Math. Soc., 19 (1988), 311-313.

[W2] __ Aspects of positively Ricci curved spaces: New examples and the fundamental group, Ph.D. thesis at SUNY at Stony Brook, 1989.

Received April 23, 1992.

THE UNIVERSITY OF MICHIGAN

ANN ARBOR, MI 48109

E-mail address: zhongmin@math.lsa.umich.edu 